\title{
QUALIDADE DE VIDA SOB A ÓPTICA DA PESSOA IDOSA INSTITUCIONALIZADA
}

\author{
Quality of life from the perspective of institutionalized older \\ people
}

\author{
Opinión del mayor institucionalizado sobre su calidad de vida
}

Artigo Original

\section{RESUMO}

Objetivo: Analisar o conceito atribuído à qualidade de vida (QV) sob a ótica de idosos institucionalizados. Métodos: Estudo descritivo, com abordagem qualitativa, realizado com oito idosas de uma Instituição de Longa Permanência (ILP) no município de Fortaleza/CE, entre setembro e outubro de 2014. As informações foram coletadas por meio de entrevista semiestruturada em encontros individuais previamente agendados, e foram seguidas as etapas de Análise de Conteúdo para organização e análise dos depoimentos. Emergiram as seguintes categorias: percepção do idoso sobre a QV; comportamentos que favorecem a QV; e concepção do idoso sobre envelhecimento saudável. Resultados: Percebeu-se que qualidade de vida envolve vários fatores objetivos e subjetivos, como vida social, cultura, saúde física e psicológica, humor e trabalho, os quais têm sido fornecidos pela Instituição de Longa Permanência onde o estudo foi realizado. A ausência de doenças foi o contraponto marcante para o conceito de envelhecer de forma saudável. Conclusão: A autonomia e a capacidade funcional estão diretamente ligadas à obtenção da qualidade de vida e ao envelhecimento saudável. Assim, a equipe multiprofissional deve promover atividades direcionadas à manutenção da funcionalidade do idoso, com o intuito de prevenir ou protelar a incapacidade, limitações e dependência da pessoa idosa.

Descritores: Qualidade de Vida; Envelhecimento; Institucionalização.

\section{ABSTRACT}

Objective: To analyze the concept of quality of life ( $Q \circ L)$ from the perspective of institutionalized older people. Methods: A qualitative descriptive study conducted with eight older women in a long-term care (LTC) institution of Fortaleza, Ceará, from September to October 2014. Data were collected through semi-structured interviews in previously scheduled individual meetings and Content Analysis steps were followed to organize and analyze the data. The following categories emerged: older people's perception of QoL; behaviors favoring quality of life; and older people's understanding of healthy aging. Results: It was found that quality of life involves various objective and subjective factors, such as social life, culture, physical and psychological health, mood, and work, which have been provided by the Long Term Care Institution where the study took place. The absence of disease was the main definition of healthy aging. Conclusion: Autonomy and functional capacity are directly linked to quality of life and healthy aging. Thus, the multidisciplinary team should promote activities aimed at maintaining the functionality of older people in order to prevent or delay disability, limitations and dependence in older people.

Descriptors: Quality of Life; Aging; Institutionalization.

\begin{abstract}
Ana Priscila Marques Lima ${ }^{(1)}$ Karen Virgínia Lopes Gomes ${ }^{(1)}$ Natasha Marques Frota ${ }^{(1)}$ Francisco Gilberto Fernandes Pereira $^{(2)}$
\end{abstract}

1) Centro Universitário Estácio do Ceará ESTÁCIO/FIC - Fortaleza (CE) - Brasil

2) Universidade Federal do Piauí - UFPI Teresina (PI) - Brasil

Recebido em: 29/01/2016 Revisado em: 16/02/2016 Aceito em: 30/03/2016 


\section{RESUMEN}

Objetivo: Analizar la opinión del mayor institucionalizado sobre el concepto de calidad de vida (CV). Métodos: Estudio descriptivo de abordaje cualitativo realizado con ocho mujeres mayores de una Institución de Larga Permanencia (ILP) del municipio de Fortaleza/CE entre septiembre y octubre de 2014. Las informaciones fueron recogidas a través de entrevista semiestruturada realizada durante encuentros individuales marcados con antelación y siguiendo las etapas del Análisis de Contenido para la organización y el análisis de los relatos. Emergierón se las siguientes categorías: percepción del mayor sobre la $\mathrm{CV}$; conductas favorables a la CV y concepción del mayor sobre el envejecimiento saludable. Resultados: Se percibió varios factores objetivos y subjetivos involucrados con la $\mathrm{CV}$ tales como la vida social, la cultura, la salud física y psicológica, el humor y el trabajo los cuales han sido fornecidos por la ILP donde se ha realizado el estudio. La ausencia de enfermedad ha sido el contrapunto principal para el concepto de envejecimiento saludable. Conclusión: La autonomía y la capacidad funcional están conectadas a la obtención de la CV y envejecimiento saludable. Así, el equipo multiprofesional debe promocionar actividades dirigidas al mantenimiento de la funcionalidad del mayor con el objetivo de prevenir o retrasar la incapacidad, las limitaciones y la dependencia de la persona mayor.

Descriptores: Calidad de Vida; Envejecimiento; Institucionalización.

\section{INTRODUÇÃO}

O Brasil é um país que envelhece a passos largos, o que é evidenciado pela mudança acelerada na estrutura etária. Em 2010, a população idosa era de 20,5 milhões, o equivalente a $10,8 \%$ da população total. Projeções indicam que, em 2020, a terceira idade brasileira será de 30,9 milhões, representando $14 \%$ da população total ${ }^{(1)}$.

Com a transição epidemiológica e as novas configurações familiares, é crescente também o número de idosos institucionalizados em vários países, inclusive no Brasil. Esse fenômeno ocorre geralmente quando o indivíduo se torna dependente e surge a necessidade de ser auxiliado por familiares ou terceiros, os quais, por sua vez, por incapacidade de cuidar, por falta de recursos ou dificuldades de encontrar cuidador, optam pela internação nas Instituições de Longa Permanência (ILP) ${ }^{(2)}$.

A institucionalização pode produzir um distanciamento progressivo da família em relação ao idoso, conduzindo em alguns casos a situações de abandono. No entanto, dependendo da estrutura, do contexto e da dinâmica familiar em que vive o idoso, a ILP assume uma função importante de reintegração social ${ }^{(3,4)}$.
As condições de vida e saúde dos idosos residentes em ILP variam de dependência total, dependência parcial e independência para a realização de atividades de vida diária. Dessa forma, a busca pela autonomia dos idosos é um ponto importante a ser discutido nas ILP, as quais devem fomentar cada vez mais atividades de promoção à saúde ${ }^{(4)}$.

Uma das teorias mais antigas e influentes sobre adaptação ao envelhecimento afirma que o bem-estar na velhice é promovido pelos altos níveis de participação em atividades sociais, atividades de lazer e mudanças de papéis $^{(5)}$.

Assim, a preocupação com a QV do idoso ganhou uma atenção maior nas ultimas décadas, devido ao aumento da expectativa de vida da população. Apesar de não existir uma definição exata sobre a $\mathrm{QV}$, tem-se utilizado a definição do World Health Organization Quality of Life Group (WHOQOL), que a define como a percepção que o indivíduo tem de sua posição na vida, no contexto da cultura e no sistema de valores em que vive, e em relação aos seus objetivos, expectativas, padrões e preocupações ${ }^{(6)}$.

Apesar das ILP atenderem às necessidades do idoso, como moradia, boa higiene, alimentação e acompanhamento médico, há uma espécie de isolamento de suas atividades familiares e sociais, vivendo muitas vezes em situações limitadas, que podem afetar a sua qualidade de vida (QV).

Frente a esse contexto, surge a necessidade de identificar a maneira como a pessoa idosa institucionalizada compreende qualidade de vida e que atividades ou comportamentos são desenvolvidos ou encorajados para o alcance desse aspecto de bem-estar.

Assim, questiona-se: qual a concepção de idosos institucionalizados sobre a qualidade de vida? Justifica-se encontrar resposta a essa pergunta para identificar áreas deficitárias que necessitem de maior estímulo organizacional e político para seu desenvolvimento, bem como descobrir capacidades individuais e coletivas no esforço pela QV em idosos institucionalizados.

O objetivo deste estudo foi analisar o conceito atribuído à qualidade de vida sob a ótica de idosos institucionalizados.

\section{MÉTODOS}

Trata-se de um estudo descritivo, com abordagem qualitativa, desenvolvido numa Instituição de Longa Permanência (ILP) onde residem 28 idosas, no município de Fortaleza/CE/Brasil. A referida ILP é gerenciada e ligada a uma entidade religiosa e proporciona moradia, cuidados com a saúde, condições de higiene e boa alimentação, além de várias outras atividades. Para admissão das idosas, os familiares entram em contato com a ILP; caso haja disponibilidade, a idosa realiza uma entrevista e o 
acolhimento. Os critérios para admissão da idosa são: ter mais de 60 anos, ser do sexo feminino, apresentar lucidez para maior desempenho das atividades e dispor de $70 \%$ do beneficio salarial de aposentadoria para manutenção do ambiente e outras necessidades.

Participaram do estudo oito idosas que atenderam aos critérios para validação das informações: idosas que residiam na instituição asilar em estudo, com quadro clínico estável, em condições cognitivas e mentais para responder o instrumento da pesquisa. Não fizeram parte do estudo as participantes que não atenderam a esses critérios; outras não tiveram interesse em contribuir com o estudo.

Quanto ao número de informantes, foi condicionado ao critério de saturação dos dados, que ocorre quando as informações tornam-se repetidas ou o acréscimo de novos dados for mínimo para submeterem-se aos procedimentos de análise ${ }^{(7)}$.

A coleta de dados ocorreu em setembro e outubro de 2014, quando foram agendados encontros individuais dos pesquisadores com os informantes para aplicação de uma entrevista semiestruturada com questões que contemplaram dados de identificação (idade, tempo de institucionalização, morbidades), fatores que interferem na qualidade de vida $\mathrm{e}$ atividades de vida diária.

Para análise das informações emergidas das entrevistas, utilizou-se a Análise de Conteúdo na modalidade temática, definida como uma técnica que consiste em apurar descrições de conteúdo aproximativas, subjetivas, para pôr em evidência a objetividade, a natureza e as forças relativas aos estímulos a que o sujeito é submetido(7). Assim, optou-se por elencar as etapas da técnica segundo o autor ${ }^{(7)}$, o qual as organiza em três fases: 1) pré-análise, 2) exploração do material e 3) tratamento dos resultados, inferência e interpretação. A pré-análise é a fase em que se organiza o material a ser analisado com o objetivo de tornálo operacional, sistematizando as ideias iniciais.

A exploração do material constitui a segunda fase, que consiste na definição de categorias (sistemas de codificação) e identificação das unidades de registro (unidade de significação a codificar corresponde ao segmento de conteúdo a considerar como unidade base, visando à categorização e à contagem frequencial) e das unidades de contexto nos documentos (unidade de compreensão para codificar a unidade de registro que corresponde ao segmento da mensagem, a fim de compreender a significação exata da unidade de registro). A exploração do material é a fase da descrição analítica, a qual diz respeito ao corpus (qualquer material textual coletado) submetido a um estudo aprofundado, orientado pelas hipóteses e referenciais teóricos.

A terceira fase diz respeito ao tratamento dos resultados, inferência e interpretação. Essa etapa é destinada ao tratamento dos resultados. Ocorre nela a condensação e o destaque das informações para análise, culminando nas interpretações inferenciais; é o momento da intuição, da análise reflexiva e crítica $^{(7)}$.

Após leitura e reflexões do conteúdo produzido através das entrevistas, surgiram três categorias: percepção do idoso sobre a QV, comportamentos que favorecem a QV e concepção do idoso sobre envelhecimento saudável.

Destaca-se que, antes de iniciada a coleta de dados, as idosas foram orientadas quanto aos objetivos do estudo e depois assinaram o Termo de Consentimento Livre e Esclarecido. O sigilo sobre as informações prestadas e a omissão de suas identidades lhes foi garantido, e à sua identidade foram atribuídos nomes de flores. Em obediência à Resolução 466/12(8), o projeto foi submetido e aprovado pelo Comitê de Ética e Pesquisa da Universidade Federal do Ceará (UFC), sob protocolo n ${ }^{\circ} 953.312$.

\section{RESULTADOS E DISCUSSÃO}

Neste espaço, serão apresentados os dados de identificação das participantes do estudo. Os resultados que se seguem exploram a subjetividade de idosas que residem em ILP acerca da QV, conforme categorias temáticas.

\section{Dados de identificação das participantes}

A princípio, demonstra-se que os dados de identificação coletados pela entrevista permitem construir o seguinte perfil das entrevistadas: oito idosas, na faixa etária de 60 a 80 anos, com média de sete anos de internas na instituição. Com relação ao quadro clínico, cinco possuíam Diabetes mellitus, e um dos motivos mais frequentes para a internação na ILP era a não disponibilidade dos familiares em exercer os cuidados com elas.

Nota-se que esse é um perfil condizente com a realidade brasileira ${ }^{(9)}$, evidenciado pelo aumento expressivo da expectativa de vida nos últimos anos, e com alta frequência de doenças endócrino-metabólicas. Considerase também que as novas configurações familiares não apresentam disponibilidade para prestação de cuidados à pessoa idosa, seguindo-se duas alternativas: o abandono ou a institucionalização responsável ${ }^{(9)}$.

\section{Percepção do idoso sobre a qualidade de vida}

Esta categoria explora as variáveis que as idosas consideraram como impactantes para atribuição do conceito de QV quando se envelhece dentro de uma ILP.

De modo geral, a percepção sobre $\mathrm{QV}$ pode ser influenciada por vários fatores que envolvem dimensões emocionais, sociais, culturais e físicas, assim, o envelhecimento pode apresentar circunstâncias que supervalorizem certas dimensões em detrimento a outras ${ }^{(10)}$. 
O envelhecimento é um processo de adaptações que sobressaltam à esfera biológica e afeta, portanto, todas as dimensões subjetivas e objetivas de vida e a autoestima da pessoa. $\mathrm{E}$, conforme o ambiente em que o indivíduo vivencia esta fase, o conceito atribuído à QV muda, sendo necessário identificar os atributos que os idosos institucionalizados determinam como importantes para o desenvolvimento desse conceito $^{(10)}$.

A avaliação da QV deve ser feita a partir de visão holística e multidimensional, sendo possível perceber a influência desses fatores através das seguintes falas:

"Qualidade de vida é ter saúde, viver em paz, sem estresse e sem brigas com as outras pessoas do abrigo." (Jasmin)

"Eu tinha uma melhor qualidade de vida quando vivia com minha família.” (Lírio)

"Para termos qualidade de vida precisamos de uma aposentadoria, pois com ela temos uma vida mais confortável. É poder realizar as próprias atividades; é sentir-se útil e ter saúde." (Íris)

As narrativas mostram que a $\mathrm{QV}$ envolve vários fatores objetivos e subjetivos, como vida social, cultura, saúde física e psicológica, humor e trabalho. É marcante na fala de Lírio a ruptura existente entre dois períodos, um quando ela residia com os familiares, e outro quando passou a morar na ILP, denotando, portanto, que o entorno e as relações familiares podem afetar no envelhecimento saudável.

No que se refere à QV para a população idosa, é importante conhecer as diferentes situações e realidades nas quais esses indivíduos podem estar inseridos. Sabe-se que quando a pessoa está vivendo com a família, muitas e enriquecedoras são as relações e trocas que ocorrem nesse ambiente e em ações na comunidade, principalmente para quem vê sua autonomia e dependência funcional ameaçadas, como é o caso da população idosa ${ }^{(11)}$.

Esse conceito, embora possa ser construído subjetivamente, está relacionado a vários fatores intrínsecos e extrínsecos, dos quais se destacam: meio ambiente, recursos econômicos, rede de relacionamentos, autonomia, independência, saúde física, tempo para o trabalho e lazer ${ }^{(12)}$. Todas essas dimensões estiveram presentes nos depoimentos dos informantes deste estudo, o que permite suspeitar que a forma como esse conceito é elaborado depende do ambiente onde a idosa reside, bem como dos níveis de importância atribuídos a cada um dos fatores.

\section{Comportamentos que favorecem a qualidade de vida}

Buscou-se nesta categoria descrever quais os comportamentos adotados pelas idosas para aumentar o seu nível se satisfação com a QV durante a institucionalização.
A transferência do lar para uma ILP pode trazer para o idoso vários conflitos na sua adaptação, pois são inegáveis as mudanças na vida ${ }^{(13)}$ durante esse processo de transição. Com o intuito de melhorar a adaptação, a ILP pode proporcionar um ambiente estimulante, permitindo a interação com outras pessoas na mesma faixa etária, uma vida ativa e independente, respeitando as restrições típicas da idade e contribuindo para melhorar sua adaptação a esse novo ambiente, longe de seus familiares.

Esse contexto traz nas falas o que as idosas fazem para alcançar seu nível de satisfação com a QV:

"Procuro fazer minhas atividades diárias, sem ajuda de outra pessoa. Isso me faz sentir bem e independente." (Jasmin)

"Cuido da minha saúde, não me entrego a doenças. Procuro fazer coisas que me deixe [m] alegre.” (Lótus)

"Faço minhas atividades diárias sem depender de ninguém, vou ao banco quando necessário. O que me deixa em paz, tranquila, de bem com a vida, é quando eu faço minhas costuras, pois sei que estou sendo útil. " (Íris)

Nesse sentido, percebe-se a importância de permitir que as pessoas idosas mantenham sua autonomia, uma vez que se sentem alegres e úteis quando realizam atividades independentes, conforme destacaram Lótus e Íris.

A independência, a autonomia, o bem-estar psicológico e o sentimento de utilidade social têm forte ligação com as dimensões da QV. Complementarmente, as atividades da vida diária (AVD), que integram a cognição, o humor, a mobilidade e a comunicação, também devem ser estimuladas, principalmente em ambientes de institucionalização( ${ }^{(9)}$.

Chama atenção que, em todos os depoimentos, não foi mencionado o ato de praticar atividades físicas, embora a ILP ofereça esse tipo de serviço, inclusive em parceria com outras instituições do terceiro setor. Ressalta-se que, embora não tenha sido destacada a regularidade da prática de exercícios ou atividade física, uma alternativa para o idoso adquirir QV está na prática de atividade física regular, a qual traz inúmeros benefícios, tanto físicos como psicossociais. Dessa forma, torna-se importante a implementação de programas que incentivem e orientem a pessoa idosa quanto à prática desse hábito ${ }^{(14)}$.

Os achados do presente estudo mostram que o idoso que reside em ILP precisa estar ativo, apto a desenvolver suas atividades de vida diária para obter $\mathrm{QV}$, o que corrobora com um estudo ${ }^{(15)}$ realizado no Rio Grande do Sul, onde o Núcleo de Atividades para Terceira Idade (NATI) promove atividade física focada na funcionalidade do idoso, com o intuito de promover saúde e propiciar um envelhecimento saudável. 
A capacidade funcional do idoso está relacionada diretamente ao seu envelhecimento ativo e bem sucedido, levando em consideração que o envelhecer saudável requer uma autonomia do idoso para a realização de sua atividade de vida diária, e para isso é necessário que o indivíduo tenha boa disposição e capacidade funcional, como é demonstrado nas falas de Jasmin e Iris.

Desse modo, entende-se que a ILP deve observar aspectos considerados relevantes na autoconcepção das idosas quanto aos atributos mais importantes para QV. No entanto, não pode se omitir de trabalhar com aspectos também cruciais, como: assistência socioeconômica e emocional, interação social, estímulo a atividades de exercícios intelectuais, incentivo ao autocuidado e suporte assistencial de saúde.

\section{Concepção do idoso sobre o envelhecimento saudável}

A QV apresenta estreita relação com a concepção de saúde integral física e mental ${ }^{(6)}$. No entanto, durante o envelhecimento, é natural que algumas funções sistêmicas corporais sofram certo declínio em virtude do próprio envelhecimento celular, o que gera muitas vezes a ideia de que envelhecer é sinônimo de adquirir doenças. Partindo desse pressuposto, esta categoria visa apresentar a visão das idosas sobre o que é envelhecer de forma saudável.

O processo de envelhecimento, na maioria das vezes, é vivenciado com certo pesar em virtude das dificuldades de lidar com essa fase de transição, o que pode desencadear doenças físicas e psicológicas ${ }^{(4)}$. É possível observar que a QV representa um fator fundamental para gerar um envelhecimento saudável.

Frente a essa relação, percebeu-se que as idosas da ILP conceituam o envelhecimento saudável, contrapondo-o a presença de doenças, explícito nos depoimentos a seguir.

"É quando a pessoa envelhece com saúde, que é quase impossivel; na verdade, é quando envelhece e não tem muitas doenças.” (Carmélia)

"Envelhecimento saudável é quando ficamos velhos sem nenhuma doença, e quando podemos fazer nossas atividades diárias sem auxilio de outra pessoa." (Íris)

"É muito dificil ficar velho sem ter nenhuma doença, mas envelhecer saudável é você cuidar da sua saúde para não ter muitas doenças." (Margarida)

Estudo realizado em Portugal afirma que o envelhecimento faz parte do ciclo de vida de todas as pessoas e provoca diversas mudanças no organismo, que devem ser encaradas como um processo natural ${ }^{(16)}$. No entanto, o decréscimo das funções fisiológicas e a resistência desse organismo devido à idade avançada causam transtornos.
A autonomia é um elemento central para o envelhecimento ativo e saudável. A promoção da autonomia e autodeterminação da pessoa idosa mantém a dignidade, integridade e liberdade de escolha, sendo essas características fundamentais para a promoção de melhores condições de saúde ${ }^{(15)}$. Enfatiza-se, ainda, a importância de promover a autonomia de idosos que residem em ILP, para que desenvolvam suas atividades de vida diária ${ }^{(4)}$.

Sabe-se que, para a redução da mortalidade e a promoção da saúde, o estilo de vida ativo é extremamente importante ${ }^{(17)}$. Os achados do presente estudo corroboram, ao afirmarem que o idoso deve procurar desenvolver algum trabalho no qual possam sentir-se úteis, aumentando, dessa forma, sua autoestima e proporcionando uma vida saudável e com qualidade.

Nesse contexto, tem-se o profissional de saúde como agente promotor da saúde, que na sua assistência ao idoso precisa trabalhar a manutenção e valorizar sua autonomia, visando construir o maior grau possível de independência funcional ${ }^{(18)}$.

Assim, para que o idoso residente da ILP consiga obter a QV, torna-se pertinente a adesão a atividades educativas, lúdicas, práticas de exercícios físicos e mentais, bem como a um acompanhamento pela equipe multiprofissional, de forma a assistir o idoso no âmbito biopsicossocial.

A disposição para realizar as atividades cotidianas, como alimentar-se sem auxílio e tomar banho sem ajuda de terceiros, é fundamental para a autonomia e independência do idoso, as quais ficam prejudicadas em virtude do aparecimento de doenças.

\section{CONCLUSÃO}

O estudo evidenciou que a independência, a autonomia, o bem-estar psicológico e o sentimento de utilidade social têm forte ligação com as dimensões da QV.

Viu-se que a saída das idosas do lar, afastando-se de seus familiares é um agravante para o envelhecimento saudável. As entrevistadas também referiram que o surgimento de doenças nessa fase da vida interfere na autonomia e independência do idoso. A fim de minimizar essa problemática, a equipe multiprofissional pode facilitar a adesão às práticas das atividades com idosos, com informações e orientações, objetivando o envelhecimento saudável.

Algumas limitações foram encontradas na realização do presente estudo, como um número pequeno de idosas que participaram, levando em consideração que os dados foram coletados apenas em uma ILP, e a participação apenas do sexo feminino, que era o perfil aceito pela ILP. Apesar das limitações encontradas, exploraram-se questões 
importantes acerca da percepção do idoso institucionalizado quanto à qualidade de vida.

Reforça-se, portanto, que, no contexto da institucionalização, a equipe multiprofissional tem como objetivo a promoção de atividades direcionadas à manutenção da funcionalidade do idoso, com o intuito de prevenir ou protelar a incapacidade, limitações e dependência da pessoa idosa.

\section{REFERÊNCIAS}

1. Malta DC, Oliveira MR, Moura EC, Silva SA, Zouain CS, Santos FP et al. Fatores de riscos e proteção para doenças crônicas não transmissíveis entre beneficiário de saúde suplementar: resultados do inquérito telefônico vigente. Ciênc Saúde Coletiva. 2011;16(3):2011-22.

2. Marin MJS, Miranda FA, Fabbri D, Tinelli LP, Storniolo LP. Compreendendo a história de vida de idosos institucionalizados. Rev Bras Geriatr Gerontol. 2012;15(1):147-54.

3. Néri AL, Yassuda MS, Fortes-Brugos AC, Mantovani EP, Arbex FS, Souza Torres SV et al. Relationships between gender, age, family conditions, physical and mental health, and social isolation of elderly caregivers. Int Psychogeriatr. 2012;24(3):472-83.

4. Alves-Silva JDA, Scorsolini-Comin F, Santos MA. Idosos em instituição de longa permanência: desenvolvimento, condições de vida e saúde. Psicol Reflex Crit. 2013;26(4):660-6.

5. Fernandez-Mayoralas G, Rojo-Perez F, MartinezMartin P, Prieto-Flores ME, Rodriguez-Blazquez, Martin-Garcia S, et al. Active ageing and quality of life: factors associated with participation in leisure activities among institutionalized older adults, with and without dementia. Aging Ment Health. 2015;19(11):1031-41.

6. Beckter M, Irigaray TQ, Trentini CM. Qualidade de vida, cognição e desempenho nas funções executivas de idosos. Estud Psicol. 2012;29(2):155-62.

7. Bardim L. Análise de conteúdo. Lisboa: Edições 70; 2010.

8. Ministério da Saúde (BR). Resolução 466/12: regulamenta os procedimentos éticos em pesquisa envolvendo seres humanos. Brasília: Ministério da Saúde; 2012.

9. Lisboa CR, Chianca TCM. Perfil epidemiológico, clínico e independência funcional de uma população idosa institucionalizada. Rev Bras Enferm. 2012;65(3):428-7.
10. Campos MO, Rodrigues JF Neto. Qualidade de vida: um instrumento para a promoção da saúde. Rev Baiana Saúde Pública. 2008;32(2):232-40.

11. Dias DSG, Carvalho CS, Araújo CV. Comparação da percepção subjetiva de qualidade de vida e bemestar de idosos que vivem sozinhos, com a família e institucionalizados. Rev Bras Geriatr Gerontol. 2013;16(1):127-38.

12. Teston EF, Marcon SS. Qualidade e condições de vida sob ótica dos residentes de um condomínio do idoso. Rev Gaúch Enferm. 2014;35(1):124-30.

13. Lima DL, Lima MAVD, Ribeiro CG. Envelhecimento e qualidade de vida de idosos institucionalizados. Rev Bras Ciênc Envelhecimento Hum. 2010;7(3);346-56.

14. Ribeiro JAB, Cavalli AS, Cavalli MO, Pogorzelski LV, Prestes MR, Ricardo LIC. Adesão de idosos a programas de atividade física: motivação e significância. Rev Bras Ciênc Esporte. 2012;34(4):969-84.

15. Borges MRD, Benedetti TRB, Rocha MFMR. Atividade física habitual e capacidade funcional percebida de idosas do sul do Brasil. Pensar Prát. 2011;14(1):1-11.

16. Santos RMF, Santos PMF, Santos VLDB, Duarte JC. A qualidade de vida do idoso: o caso de Cova da Beira. Referência. 2013;3(11):37-48.

17. Fortes LM, Sousa LEN, Fernandes MA. Atividade ocupacional como fator de qualidade de vida na terceira idade. Rev Interdisciplinar NOVAFAPI. 2011;4(4):4954.

18. Rinaldi FC, Campos MEC, Lima SS, Sodré FSS. O papel da enfermagem e suas contribuições para a promoção do envelhecimento saudável e ativo. Rev Eletrônica Gestão \& Saúde. 2013;4(2):454-66.

\section{Endereço do primeiro autor:}

Ana Priscila Marques Lima

Centro Universitário Estácio do Ceará - Unidade Via Corpvs

Rua Eliseu Uchoa Becco, 600

Bairro: Guararapes

CEP 60810-270 - Fortaleza - CE - Brasil

E-mail: anapriscil@yahoo.com.br

\section{Endereço para correspondência:}

Francisco Gilberto Fernandes Pereira

Rua Professor Vicente Silveira, 100/ 404 - Bloco 2

Bairro: Vila União

CEP: 60410-322 - Fortaleza - CE - Brasil

E-mail: gilberto.fp@hotmail.com 